\title{
Repenser le développement en observant le travail des groupes communautaires : le cas des groupes communautaires au centre urbain de Chicoutimi
}

\author{
Suzanne Tremblay \\ Université du Québec à Chicoutimi
}

\section{Introduction}

Les réflexions que nous présentons dans ce texte ${ }^{1}$ portent principalement sur le sens et sur l'interprétation de la notion de développement. Ces interprétations sont liées à l'avènement des premières thèses sur l'évolution naturelle et sur la croissance économique élaborées par les économistes classiques, ainsi que les thèses plus récentes sur le concept de développement conçues dans le cadre des programmes de développement et mises en œuvre dans les pays du tiers-monde.

Ces réflexions s'alimentent de plusieurs sources, dont le courant critique sur la mise en œuvre et la théorisation d'un post-développement suite au constat de l'échec de la notion de développement, notamment dans les pays du tiers monde $^{2}$. Notre réflexion est alimentée aussi par l'approche écologique qui remet en cause les modes de développement actuels par le courant du développement communautaire qui met au premier plan les acteurs de la communauté comme des acteurs de développement, et par le courant du développement endogène qui prend en compte les ressources et les particularités locales pour la mise en œuvre du développement ainsi que ceux du développement social, du développement humain et de l'approche féministe du développement.

À l'aide de ces théories, nous proposons d'analyser l'action des groupes communautaires à Chicoutimi dans la perspective d'une nouvelle façon de penser le développement.
Dans un premier temps, nous allons regarder quelques-uns des courants théoriques mentionnés précédemment. Par la suite, nous regarderons le cas de certains groupes communautaires à Chicoutimi et le sens à donner à leurs actions dans la recherche d'un autre développement.

\section{Les sources du cadre conceptuel du développement}

Si nous avançons la nécessité de repenser ou de redéfinir le développement aujourd'hui, c'est que le développement a déjà été pensé par nombre d'auteurs qui ont donné des définitions et inscrit cette notion dans un cadre conceptuel et théorique. Alors, en regardant les origines de ce cadre conceptuel, il nous semble que nous voyons apparaître trois éléments importants :

- les thèses évolutionnistes liées à l'évolution naturelle où le développement est assimilé au processus qui induit le changement dans l'évolution naturelle;

- les thèses économiques où les grands principes économiques ont été liés au développement. Ainsi, tant dans sa forme que dans son essence, le développement deviendra synonyme de développement économique. Cette idée de développement lié à l'économie s'imposera à nous depuis le mercantilisme en passant par la révolution industrielle et ce, jusqu'à l'époque de mondialisation et de globalisation que nous connaissons aujourd'hui; 
- le développement comme programme de mise à niveau des sociétés du tiers-monde.

Selon Gilbert Rist, « le développement occupe au sein de l'idéologie naturaliste une place à part, car il renvoie à une longue tradition qui s'étend sur la longue durée de l'histoire occidentale ${ }^{3}$. Une histoire qui va d'Aristote jusqu'au $\mathrm{XIX}^{\mathrm{e}}$ siècle où l'on verra «le triomphe de l'évolutionnisme social » où les progrès de la technique et de la science vont devenir les moteurs de la croissance et de sa représentation, soit le développement. Dans cette perspective, le développement est non seulement irréversible, mais il apparaît aussi inévitable, tout comme l'évolution naturelle.

Le concept de développement semble donc être une donnée naturelle qu'il faut mettre en œuvre d'une façon ininterrompue. Ces principes apparaissent tellement évidents et naturels que peu de gens cherchent à les questionner et à les remettre en cause. Comment, en effet, remettre en cause l'évidence et le principe de la nature elle-même?

\section{Aujourd'hui, la crise du développement s'incarne dans un clivage de plus en plus grand entre les nantis et les pauvres des sociétés du Nord comme du Sud.}

\section{La crise du développement}

Pourtant, dans le long parcours du développement, plusieurs auteurs ont fait le constat de l'échec du développement ${ }^{4}$. Ce constat s'est effectué peu à peu avec la prise de conscience de l'inégalité de la diffusion du développement dans l'espace. Autrement dit, les théoriciens du développement se sont aperçus que le développement se ne diffusait pas de façon uniforme dans l'espace. Cette prise de conscience s'est élaborée d'abord au plan international lorsqu'il est devenu évident qu'il y avait des déséquilibres de développement entre les pays du Nord et les pays du Sud. Par la suite, les analystes du développement ont constaté que des déséquilibres se produisaient aussi à l'intérieur des territoires des pays dits développés.

Aujourd'hui, la crise du développement s'incarne dans un clivage de plus en plus grand entre les nantis et les pauvres des sociétés du Nord comme du Sud, entre les inclus de ce système mondial, les manipulateurs de symboles, comme les nomment Robert Reich ${ }^{5}$ et les exclus de cette économie-monde, bref entre un monde d'opulence dont les acteurs font partie du système d'échange mondial et un monde d'exclusion où la pauvreté et la marginalité sont omniprésentes. Il se dessine ainsi des espaces d'exclusion, des territoires de la marginalisation. Ces territoires et ces espaces ne sont pas excentriques à l'économie-monde; ils se découpent à l'intérieur de chaque société de l'espace mondialisé. Ces espaces d'exclusion prennent de multiples formes, que ce soit des pays entiers en proie à la famine, par exemple dans la région de l'Afrique subsaharienne, des régions rurales ou des espaces centraux urbains en dévitalisation, etc. Ils sont à la fois des espaces géographiques mais aussi des espaces sociaux où la pauvreté, l'exclusion, la marginalité, l'excentricité deviennent les lieux communs de ces « régions qui perdent» de l'économiemonde. Ces territoires d'exclusion représentent à la fois l'impasse du développement et l'espoir d'un changement social.

\section{À la recherche d'un autre développement}

Si le développement est le fruit d'une théorisation, d'un système d'idées et d'un construit social, il est sans doute possible de penser autrement le développement et de chercher à redéfinir le contenu du concept de développement. Actuellement, plusieurs auteurs travaillent à une nouvelle théorisation du concept de développement, ou plutôt du post-développement. Ainsi, selon Gilbert Rist « la théorisation de l'aprèsdéveloppement est une tâche essentielle aujourd'hui » ${ }^{6}$.

Notre réflexion s'inspire de cette recherche d'un autre développement ou d'un post-développement. Pour ce faire, nous avons choisi d'observer à micro-échelle celle des quartiers du centre urbain de Chicoutimi les groupes communautaires qui travaillent depuis quarante ans sur ce territoire. Nous nous sommes demandée si l'action des groupes communautaires qui œuvrent au centre urbain de Chicoutimi allait dans le sens de cette recherche d'un autre développement. Avant de tenter de répondre à cette question, nous allons regarder brièvement la problématique de pauvreté relative au territoire du centre urbain de Chicoutimi. 


\section{Le territoire du centre-ville de Chicoutimi}

Comme plusieurs centres urbains dans les villes de l'Amérique, du Canada, du Québec et même de l'Europe, celui de Chicoutimi est un territoire marqué par la pauvreté et la désintégration. Il faut remonter à la petite histoire du développement de la ville pour comprendre ce qui a amené cette situation où l'on retrouvait, en 1996, $45 \%$ de personnes à faible revenu dans le quartier centre-ville lui-même, et de $30 \%$ à $35 \%^{7}$ de personnes à faible revenu dans les autres quartiers du centre urbain de la ville. Il s'agit en fait d'un long processus de dévitalisation qui a pris naissance à la fin des années 1960 dans le sillage de la modernisation qui était mise en œuvre à Chicoutimi, comme ailleurs au Québec. La construction du pont Dubuc et de ses voies d'accès au cœur des quartiers centraux, les nombreuses démolitions de maisons dans les quartiers centraux, sans véritable programme de reconstruction ainsi que le déplacement des populations dans les quartiers attenants aux centres commerciaux et aux quartiers dortoirs de la ville apparaissent parmi les facteurs les plus importants de cette dévitalisation.

Ainsi, cette dévitalisation, qui a débuté dans les années 1970, a continué à s'accélérer au fil des décennies et, à la fin des années 1990, la situation au centre urbain de Chicoutimi ne s'est guère améliorée. Il semble donc y avoir une portion de la population à Chicoutimi qui ne puisse jouir des fruits du développement basé sur la croissance économique productiviste.

De plus, cette portion de la population semble se territorialiser dans certains territoires en mal de développement, car la pauvreté n'est pas abstraite et elle s'incarne dans des territoires qui sont laissés pour compte par la diffusion inégale du développement. Cela montre que l'inégalité du développement semble toucher d'abord certaines classes sociales, et les inégalités sociales tendent à se territorialiser.

Il paraît donc y avoir un lien entre les inégalités sociales et les territoires qui sont en dehors de la trajectoire du développement. Ainsi, ces territoires du non-développement sont en même temps des espaces sociaux qui s'incarnent dans le territoire des villes et des régions. Les centres urbains comme celui de Chicoutimi font partie de ces espaces. De plus, si la situation du centre urbain de Chicoutimi apparaît aus- si désastreuse, il faut considérer qu'il s'agit ici d'un centre urbain d'une région ressource, une région qui subit elle-même la dévitalisation et la désintégration. Car si le développement tend à se polariser dans l'espace, le non-développement semble se territorialiser lui aussi dans les espaces laissés pour compte. Une théorie du développement ${ }^{8}$ postule que la polarisation du développement entraîne la croissance économique pour les espaces en périphérie. Mais se pourrait-il que la polarisation du développement amène plutôt le non-développement des espaces périphériques?

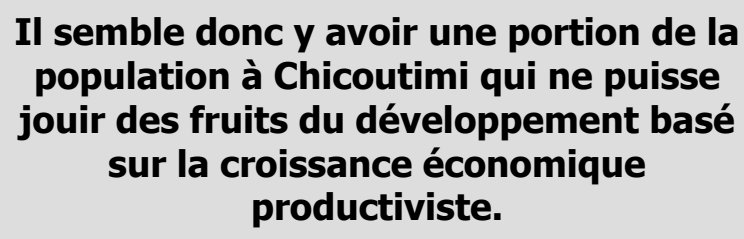

La question que nous nous sommes posée est de savoir si, dans ces territoires laissés pour compte, il pouvait y avoir un autre développement. Car le développement dont nous avons parlé jusqu'à maintenant est toujours le développement classique basé sur la croissance économique. Mais se peut-il qu'il existe une autre forme de développement? Qui ne soit pas basée uniquement sur la croissance économique? C'est ce que nous avons voulu observer en regardant l'expérience des groupes communautaires qui œuvrent dans le centre urbain de Chicoutimi depuis au moins quarante ans.

\section{À la recherche d'un autre développement ?}

Pour tenter de répondre à cette question, nous avons entrepris l'étude de l'action de certains groupes communautaires œuvrant sur le territoire du centre urbain de Chicoutimi.

Parmi les groupes étudiés, il y a celui de l'action catholique. Il ne s'agit pas d'un seul groupe mais plutôt d'une famille de groupes rassemblés sous le chapeau de la centrale de l'action catholique qui a pignon sur rue au centre-ville de Chicoutimi depuis environ quarante ans. Nous avons observé le travail des groupes d'action catholique depuis 1960 jusqu'à aujourd'hui. $\mathrm{Au}$ fil de ces quatre décennies, nous avons pu observer l'évolution du travail de ces groupes. Dans les années 1960, les militants des groupes d'action catho- 
lique à Chicoutimi ont été, selon une étude effectuée sur les pratiques en travail social au Saguenay-LacSt-Jean ${ }^{9}$, parmi les premiers travailleurs communautaires au Saguenay-Lac-Saint-Jean. Dès cette époque, les préoccupations d'ordre social et de « justice redistributive $\gg{ }^{10}$ étaient présentes dans les actions des groupes d'action catholique. Cependant, c'est véritablement dans les années 1970 que ces groupes ont développé une action plus revendicative axée vers le changement social et la lutte pour les personnes appauvries de la société. À cette époque, les groupes d'action catholique commencèrent à s'allier aux autres groupes populaires et communautaires dans les manifestations et les diverses luttes des travailleurs, des chômeurs et des assistés sociaux. Cette action pour les personnes défavorisées prit une allure encore plus concrète dans la décennie 1980 alors que les groupes d'action catholique contribuèrent à la mise sur pied de nombreux organismes pour aider les jeunes et les personnes appauvries. En effet, bien que leur présence soit discrète, comme ils l'affirment, leur action est tangible sur le territoire du centre urbain puisqu'ils ont permis de voir apparaître dans le paysage du centre-ville un comptoir vestimentaire, une cuisine collective, un service de travailleurs de rue, un Café jeunesse et une épicerie populaire, etc.

Et puis, dans le milieu des années 1980 et dans la décennie 1990, c'est l'économie solidaire et l'intervention de quartier qui devinrent les nouvelles formes d'action des groupes d'action catholique Dans ce sillage naquirent un carrefour communautaire de quartier, un collectif de micro-entreprises et la transformation du presbytère du Bassin en lieu pour les groupes communautaires et les groupes liés à l'économie sociale.

\section{Et le développement dans tous cela ?}

Dans l'étude que nous avons faite, nous pouvons distinguer deux façons d'appréhender le développement. En effet, nous pouvons constater, dans un premier temps, que les nombreuses actions mises en œuvre par les groupes d'action catholique, en termes de services et d'entreprises collectives en économie sociale, s'inscrivent dans une démarche de développement communautaire à l'échelle locale.

Cependant, en analysant le discours des militants que nous avons interviewés, cela nous amène à une autre façon d'appréhender et de penser le développement. En effet, il s'agit non seulement de voir les actions concrètes de développement mises de l'avant par les groupes communautaires, mais surtout de percevoir comment ils envisagent leurs actions en termes de développement. Sur ce plan, les propos des informateurs consultés sont très révélateurs et il nous semble qu'il y a là matière à concevoir le développement d'une façon différente.

Ainsi, pour les groupes d'action catholique, le développement est d'abord celui des personnes, de réponse à leurs besoins fondamentaux de survie, mais aussi de valorisation et d'estime de soi. Ensuite, il y a un élargissement vers la communauté, la vie collective, car dans la vision des groupes d'action catholique, la communauté et le milieu sont aussi des éléments fondamentaux. La personne doit, en effet, être en interaction avec son milieu et sa communauté pour susciter une prise en charge, une amélioration de ses conditions de vie et de celles de sa communauté ou son milieu de vie. Il y a ici un lien étroit entre l'individu et la vie collective.

\section{Dans l'étude que nous avons faite, nous pouvons distinguer deux façons d'appréhender le développement.}

Par la suite, les groupes d'action catholique parlent de la notion de développement solidaire où le développement est d'abord la prise en charge des personnes et des communautés, notamment par la création d'emplois et la création de groupes de services qui deviennent, dans cette optique, des outils pour mettre en œuvre le développement à la fois des personnes et des communautés. Cela s'effectue entre autres par la prise en charge, la valorisation et l'amélioration des conditions de vie des personnes. L'idée de progrès social apparaît très importante dans cette vision ou cette perception du développement. Nous retrouvons aussi, dans cette conception du développement, la concertation des acteurs au plan local pour la mise en œuvre du développement solidaire.

Voilà donc autant d'éléments qui nous permettent d'envisager le développement d'une autre manière : la prise en compte des personnes, non seulement pour améliorer les conditions de vie aux plans de l'habitation, de la nourriture, des vêtements, mais aussi aux 
plans de la valorisation des personnes, de leur parcours, de l'expérience vécue, de l'estime de soi et de l'intégration dans la communauté. La qualité de vie comme élément central du développement est aussi une donnée fondamentale pour concevoir un autre développement. Enfin, l'entrepreneuriat collectif qui peut être associé ici à l'économie sociale, l'économie solidaire, l'autogestion, etc. est un élément qui peut permettre à la fois de créer une certaine richesse collective et de favoriser l'émergence et/ou la consolidation du lien social.

Tous ces éléments que sont la valorisation des personnes, la mise en valeur des communautés, la recherche de la qualité de vie et la promotion de l'entrepreneuriat collectif existent déjà dans les multiples courants et théories du développement qui ont cours depuis les trente dernières années. Nous n'avons qu'à penser au développement endogène, au développement durable, au développement local, à l'écodéveloppement, au développement communautaire, etc. qui sont autant de versions de la recherche de nouveaux modes de développement.

\section{La qualité de vie comme élément central du développement est aussi une donnée fondamentale pour concevoir un autre développement.}

Quant à savoir si l'on peut inscrire les actions et les préoccupations des groupes communautaires dans cette recherche d'un post-développement, il faut comprendre que, même si les préoccupations des groupes communautaires apparaissent souvent d'ordre pragmatique, elles s'inscrivent tout de même dans la recherche ou dans la quête du changement social qui peut prendre la forme d'un autre développement. La quête du changement social apparaît en effet comme une préoccupation de longue date pour les groupes communautaires, même si cette recherche a pu se transformer et prendre d'autres formes au cours des dernières décennies. De plus, les groupes communautaires sont sans doute les acteurs les plus près de cette mise en œuvre d'un autre développement puisqu'étant à proximité du terrain, ils sont les plus susceptibles de développer des pratiques novatrices pour répondre aux besoins des personnes laissées pour compte par le développement actuel.

\section{Conclusion}

La question du développement et de sa reformulation pour la prise en compte de ceux et celles qui sont laissées pour compte par le développement néo-libéral apparait comme une tâche cruciale au moment où la pauvreté s'étend partout dans le monde et à mesure que la concentration de la richesse mondiale s'accentue. À l'exemple de plusieurs auteurs du courant post-développementiste, nous pensons que les actions des groupes communautaires peuvent être porteuses d'enseignement et de pratiques novatrices pour penser et mettre en œuvre un autre développement. Ce texte propose quelques pistes de réflexion à ce sujet, mais plusieurs enseignements restent encore à tirer de l'expérience chicoutimienne.

\section{Notes et références}

1 Ces réflexions proviennent largement du questionnement de notre thèse de doctorat qui est présentement en évaluation.

2 Latouche, Serge (1991). La planète des naufragés. Essai sur l'après-développement, Paris, Éditions La Découverte, $235 \mathrm{p}$.

Rist, Gilbert (1996). Le développement: histoire d'une croyance occidentale, Paris, Presses de la Fondation nationale des sciences politiques.

Sachs, Wolfgang (1996). « Le développement : une idéologie en ruine ", dans W. Sachs et G. Esteva, Des ruines $d u$ développement, Montréal, Écosociété, p. 13-86.

Wallerstein, Immanuel (2001). «Le système-monde en désagrégation », dans D. Mercure (dir.), Une sociétémonde? Les dynamiques sociales de la mondialisation, Sainte-Foy, PUL, p. 35-44.

Wallerstein, Immanuel (1982). « La crise comme transition », dans La crise, qu'elle crise ?, Paris, Maspéro, p. 1057.

3 Rist, op. cit., p. 52.

4 Partant, François (1982). La fin du développement. Naissance d'une alternative?, Paris, Maspéro, 181 p.; Latouche, op. cit.; Sachs, op. cit.

5 Reich, Robert (1993). L'économie mondialisée, Paris, Dunod, $336 \mathrm{p}$.

6 Rist, op. cit., p. 402.

7 Tremblay, Suzanne (2004). À la recherche d'un autre développement? Dévitalisation urbaine et revitalisation 
communautaire au centre urbain de Chicoutimi, de 1960 à nos jours, Chicoutimi, thèse de doctorat, Université du Québec, à paraître, p. 226.

8 Perroux, François J. (1985) cité dans P. Aydalot, Économie régionale et urbaine, Paris, Économica. p.139.
9 St-Onge, Jacques (1999). Le développement des pratiques en travail social au Saguenay-Lac-Saint-Jean, Chicoutimi, GRIR-UQAC, $252 \mathrm{p}$.

10 Tiré des propos d'un des informateurs interviewés pour notre thèse de doctorat. 